\title{
OPEN The efficacy and safety of Favipiravir in treatment of COVID-19: a systematic review and meta-analysis of clinical trials
}

\author{
Soheil Hassanipour $\mathbb{1}^{1,6}$, Morteza Arab-Zozani ${ }^{2,6 \bowtie}$, Bahman Amani ${ }^{3}{ }^{3}$, \\ Forough Heidarzad ${ }^{1}{ }^{1}$, Mohammad Fathalipour $\mathbb{1}^{4}$ \& Rudolph Martinez-de-Hoyo ${ }^{5}$
}

The novel coronavirus outbreak began in late December 2019 and rapidly spread worldwide, critically impacting public health systems. A number of already approved and marketed drugs are being tested for repurposing, including Favipiravir. We aim to investigate the efficacy and safety of Favipiravir in treatment of COVID-19 patients through a systematic review and meta-analysis. This systematic review and meta-analysis were reported in accordance with the PRISMA statement. We registered the protocol in the PROSPERO (CRD42020180032). All clinical trials which addressed the safety and efficacy of Favipiravir in comparison to other control groups for treatment of patients with confirmed infection with SARS-CoV2 were included. We searched electronic databases including LitCovid/ PubMed, Scopus, Web of Sciences, Cochrane, and Scientific Information Database up to 31 December 2020. We assessed the risk of bias of the included studies using Cochrane Collaboration criteria. All analyses were performed using the Comprehensive Meta-Analysis software version 2, and the risk ratio index was calculated. Egger and Begg test was used for assessing publication bias. Nine studies were included in our meta-analysis. The results of the meta-analysis revealed a significant clinical improvement in the Favipiravir group versus the control group during seven days after hospitalization $(R R=1.24,95 \% \mathrm{Cl}: 1.09-1.41 ; \mathrm{P}=0.001)$. Viral clearance was more in 14 days after hospitalization in Favipiravir group than control group, but this finding marginally not significant $(\mathrm{RR}=1.11,95 \% \mathrm{Cl}$ : 0.98-1.25; $\mathrm{P}=0.094)$. Requiring supplemental oxygen therapy in the Favipiravir group was $7 \%$ less than the control group, $(R R=0.93,95 \% \mathrm{Cl}: 0.67-1.28 ; \mathrm{P}=0.664)$. Transferred to ICU and adverse events were not statistically different between two groups. The mortality rate in the Favipiravir group was approximately $30 \%$ less than the control group, but this finding not statistically significant. Favipiravir possibly exerted no significant beneficial effect in the term of mortality in the general group of patients with mild to moderate COVID-19. We should consider that perhaps the use of antiviral once the patient has symptoms is too late and this would explain their low efficacy in the clinical setting.

The novel coronavirus (SARS-CoV-2) outbreak began in late December 2019 and rapidly spread worldwide, critically impacting public health systems ${ }^{1}$. As of May 5, 2021, the Johns Hopkins Coronavirus Resource Center has reported 161,288,384 confirmed Global COVID-19 cases and a total of 3,347,154 worldwide deaths ${ }^{2}$. The clinical characteristics of Coronavirus disease 2019 (COVID-19) include respiratory symptoms, fever, cough, dyspnea, and pneumonia. In the absence of any established pharmacological agents, supportive care remains the cornerstone of clinical management for COVID-193.

As of October 22, 2020, remdesivir, an antiviral agent, is the only drug approved for treatment of COVID$19^{4,5}$. An emergency use authorization (EUA) for convalescent plasma was announced on August 23, 20206. The Food and Drug Administration (FDA) issued an EUA for bamlanivimab on November 9, 20207. An EUA was issued for baricitinib on November 19, 2020 for use, in combination with remdesivir ${ }^{8}$, and for casirivimab and

\footnotetext{
${ }^{1}$ Gastrointestinal and Liver Diseases Research Center, Guilan University of Medical Sciences, Rasht, Iran. ${ }^{2}$ Social Determinants of Health Research Center, Birjand University of Medical Sciences, Birjand, Iran. ${ }^{3}$ Department of Health Management and Economics, School of Public Health, Tehran University of Medical Sciences, Tehran, Iran. ${ }^{4}$ Department of Pharmacology and Toxicology, Faculty of Pharmacy, Hormozgan University of Medical Sciences, Bandar Abbas, Iran. ${ }^{5}$ MSN Labs Americas, Bogotá, Colombia. ${ }^{6}$ These authors contributed equally: Soheil Hassanipour and Morteza Arab-Zozani. ${ }^{\circledR}$ email: arab.hta@gmail.com
} 
imdevimab on November $21^{9}$. On December 11, 2020 the first vaccine (BNT-162b2 SARS-CoV-2 vaccine) was granted an EUA by the FDA and the same was accepted for a second vaccine (mRNA-1273 SARS-CoV-2 vaccine) on December 18, $2020^{10}$.

Numerous collaborative efforts to discover and evaluate effectiveness of antivirals, immunotherapy, monoclonal antibodies (at least 327 treatments under investigation), and 257 vaccines have rapidly emerged according to "The Milken Institute" that maintains a detailed COVID-19 Treatment and Vaccine Tracker of research and development progress ${ }^{11}$. Due to the urgency of the situation, a number of already approved and marketed drugs are being tested for repurposing, including Favipiravir ${ }^{12}$.

Favipiravir, also known as T-705, a purine nucleic acid analog, is one of the antiviral candidates considered in several clinical trials. It is a chemical used experimentally and was created by the Japanese company Toyama, a subsidiary of Fuji Film, as reported initially by Furuta in $2002^{13}$. In 2014 , it was approved in Japan as a backup choice for resistant influenza infection and since then have been approved in several countries and is indicated for the treatment of patients with mild to moderate COVID-19 disease ${ }^{14}$. Favipiravir is an RNA-dependent RNA polymerase inhibitor. It is activated in its phosphoribosylated form (Favipiravir-RTP) in cells, inhibiting viral RNA polymerase activity ${ }^{15}$.

As of the 12th October 2020, there are 37 studies registered in the ClinicalTrials.gov database to evaluate the utility of this repurposed drug to fight against COVID-19 16.

Even though multiple articles about Favipiravir are readily available for download online, including some systematic reviews and meta-analyses conducted on only two RCTs at this time ${ }^{17-21}$, the scientific community may find it challenging to get an overview regarding the safety and efficacy of this drug. Therefore, we aim to provide this systematic review and meta-analysis of Favipiravir. To do so, we assess all available completed clinical trials till December $2020^{3}$.

\section{Methods}

Protocol and registration. This systematic review and meta-analysis were reported in accordance with the Preferred Reporting Items for Systematic Reviews and Meta-Analyses (PRISMA) statement ${ }^{22}$. We registered the protocol in the International Prospective Register of Systematic Reviews (PROSPERO) (CRD42020180032). Also, we published this protocol in the BMJ Open journal ${ }^{3}$.

Eligibility criteria. All clinical trials (study design) which addressed the safety and efficacy of Favipiravir (intervention) in comparison to other control groups (comparison) for treatment of patients with confirmed infection with SARS-CoV2 (population) were included. There were no restrictions concerning gender, age, ethnicity, blinding, follow-up, or publication status. All publications in English and Farsi were included. The investigated outcomes include clinical improvement based on the WHO Ordinal Scale ${ }^{23}$, viral clearance (negative SARS-CoV-2 PCR test in nasopharyngeal and/or oropharyngeal swabs on seven, ten, or 14 days after hospitalization), transferred to ICU (need to admit in the Intensive Care Unit), supplemental oxygen therapy (need to use of oxygen to improve low $\mathrm{O}_{2}$ saturation), adverse events, and mortality. It should be noted that some of the outcomes mentioned in the protocol were not analyzed due to the lack of sufficient data in the final included articles. The data containing at least one Favipiravir-related outcome or side effects were considered sufficient. Articles with unavailable full text in English or Farsi languages or whose full text is not accessible were excluded from the study. The studies with insufficient or incomplete data were not being incorporated.

Information sources and search strategy. Two independent reviewers (MA-Z and SH) searched electronic databases including LitCovid/PubMed ${ }^{24}$, Scopus, Web of Sciences, Cochrane, and Scientific Information Database (SID) ${ }^{25}$ using keywords combination (MeSH term and free term), such as "2019 nCoV" OR 2019nCoV OR "2019 novel coronavirus" OR COVID-19 OR "new coronavirus" OR "novel coronavirus" OR "SARS CoV-2" OR (Wuhan AND coronavirus) OR "SARS-CoV" OR "2019-nCoV" OR "SARS-CoV-2" and Favipiravir OR Avigan up to 31 December 2020. We also searched two preprint databases including MedRxiv and Research Square and, the reference lists of all included studies, reviews, and clinical trial registries, for an ongoing clinical trial. In addition, we created an alarm on Google Scholar and included new related articles. (see Supplementary file 1 for the final proposed PubMed search strategy).

Study records. Once the records have been imported to EndNote X7 software and all duplicates have been removed, two reviewers (SH and BA) manually and independently screened titles, abstracts, and full-texts of included studies based on predefined eligibility criteria to identify studies concerning the safety and efficacy of Favipiravir among patients with COVID-19. All potentials discrepancies were resolved upon consultation with a third reviewer (MA-Z).

Data extraction and data items. Two reviewers ( $\mathrm{SH}$ and BA) independently extracted data from included studies, using a pre- piloted data extraction form. We piloted this form using at least three examples of included studies and if there is a $90 \%$ and above agreement, it is approved. The data extraction form includes the following items; authors name, year of the publication, study design, study sample, country of origin, mean age of participants, gender, the severity of diseases, comorbidities, type of intervention and dose, control group, follow up, randomization, blinding, allocation concealment, primary and secondary outcomes, and adverse events $^{3,26}$. All potentials discrepancies were resolved by consultation with a third reviewer (RM). 


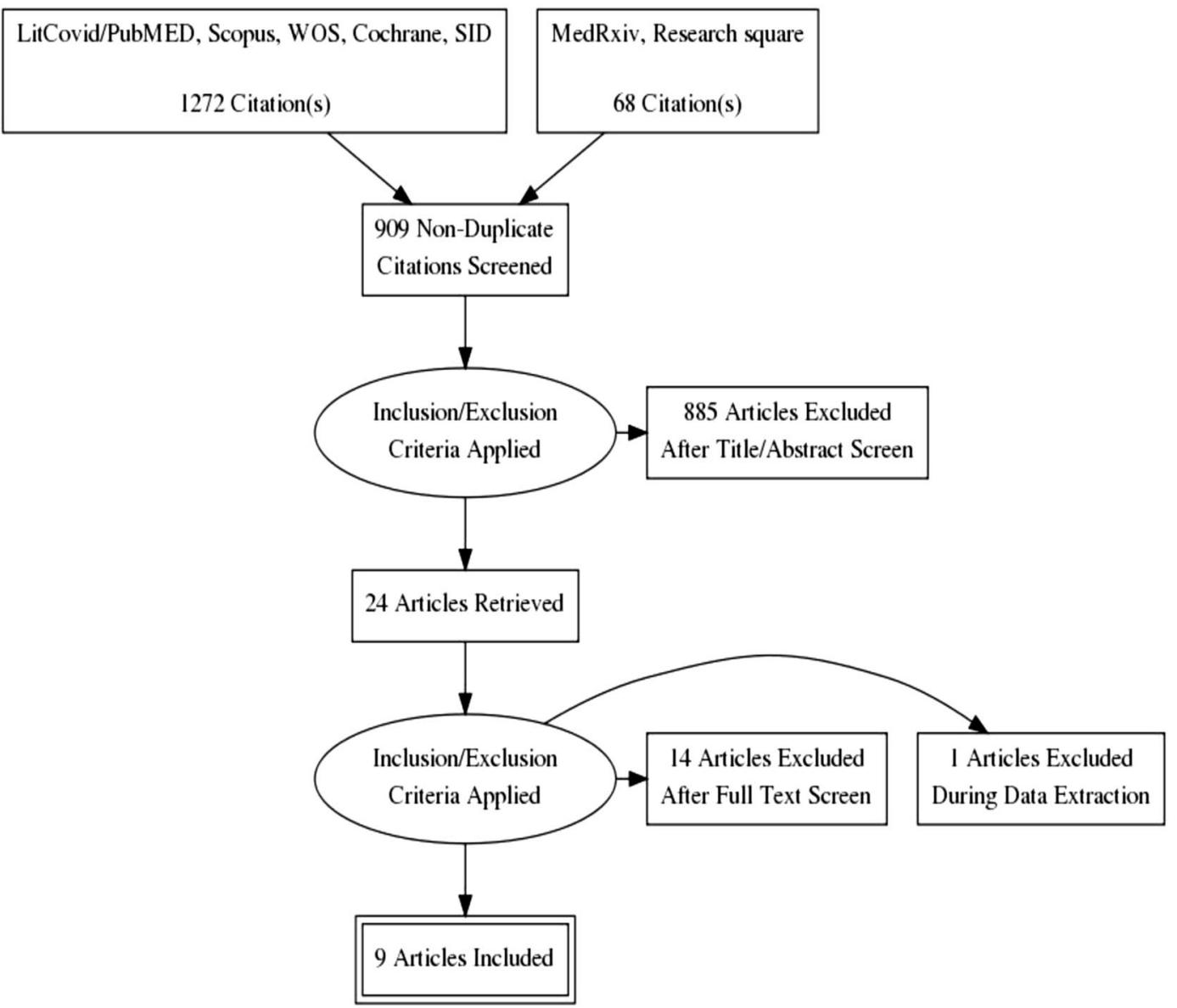

Figure 1. Search process and study flow diagram.

Risk of bias in individual studies. Two reviewers (MF and $\mathrm{FH}$ ) independently assessed the risk of bias among the included studies. We assessed the risk of bias of the included studies using Cochrane Collaboration criteria, including seven items of selection bias (random sequence generation and allocation concealment), performance bias, detection bias, attrition bias, reporting bias, and other forms of bias ${ }^{3,26}$. Any discrepancies were resolved upon consultation with a third reviewer (MA-Z).

Statistical analysis. All analyses were performed using the Comprehensive Meta-Analysis (CMA; Borenstein, Hedges, Higgins, and Rothstein) software version 2, and the risk ratio (RR) index was calculated. CMA software has the ability to combine different indices and to combine the effect of sample size and the difference of the index being compare ${ }^{27}$. We used the $\mathrm{I}^{2}$ statistics and Cochran test (with significantly less than 0.1 ) to assess the heterogeneity of the included studies ${ }^{28}$. In cases where there was heterogeneity, we performed the randomeffect model. We also used a subgroup analysis based on follow up days for clinical improvement and viral clearance. One-leave-out sensitivity analysis were conducted for all outcomes based on Cochrane recommendation. Egger and Begg test was used for assessing publication bias.

\section{Results}

Description of search. We identified a total of 1340 records after searching the databases. After the removal of 431 duplicate records, the title and abstracts of 909 records were screened. Eight hundred eighty-five records were excluded after title and abstracts screening, and 24 records were assessed for full-text screening. A total of 15 records were excluded based on eligibility criteria. The main reasons for the exclusion included inadequate information $(n=7)$, absence of study outcome $(n=6)$, and absence of control group $(n=2)$. Finally, nine studies were included in our meta-analysis ${ }^{29-37}$ (Fig. 1).

Characteristics of the included studies. Nine studies encompassing 827 patients were included. According to the geographical area, four studies were conducted in China (44.4\%), and Russia, Oman, Egypt, and Japan also had an article. Only one study was nonrandomized. The minimum follow-up time was 10 days, and the maximum was 30 days. The doses of Favipiravir and control drugs in each study were different. All studies registered in clinical trial registries. The summary characteristics of the included studies have been summarized in Table 1. 


\begin{tabular}{|c|c|c|c|c|c|c|c|c|c|c|}
\hline Authors/year & \begin{tabular}{|l|} 
ClinicalTrials \\
identifier
\end{tabular} & $\begin{array}{l}\text { Publication } \\
\text { status }^{\mathrm{a}}\end{array}$ & Journal & Country & Study design & Age & Male & $\begin{array}{l}\text { Intervention/ } \\
\text { sample size }\end{array}$ & $\begin{array}{l}\text { Comparison/ } \\
\text { sample size }\end{array}$ & Follow up \\
\hline $\begin{array}{l}\text { Cai } \\
\text { et al./2020 }\end{array}$ & ChiCTR2000029600 & In press & Engineering & China & $\begin{array}{l}\text { Open-label, } \\
\text { nonran- } \\
\text { domized, } \\
\text { before-after } \\
\text { controlled } \\
\text { study }\end{array}$ & $16-75$ & \begin{tabular}{|l|}
$40 \%$ in FVP \\
group/46.7\% \\
in LPV/RTV \\
group
\end{tabular} & $\begin{array}{l}\text { Oral FPV (Day } \\
\text { 1: } 1600 \mathrm{mg} \\
\text { twice daily; } \\
\text { Days 2-14: } 600 \\
\text { mg twice daily) } \\
\text { plus interferon } \\
\text { (IFN)-a by aero- } \\
\text { sol inhalation (5 } \\
\text { million U twice } \\
\text { daily)/35 }\end{array}$ & \begin{tabular}{|l|} 
LPV/RTV (Days \\
1-14: $400 \mathrm{mg} / 100$ \\
mg twice daily) \\
plus IFN-a by \\
aerosol inhala- \\
tion ( 5 million U \\
twice daily)/45
\end{tabular} & Day 14 \\
\hline $\begin{array}{l}\text { Chen } \\
\text { et al./2020 }\end{array}$ & ChiCTR2000030254 & Preprint & MedRxiv & China & $\begin{array}{l}\text { Prospective, } \\
\text { randomized, } \\
\text { controlled, } \\
\text { open-label } \\
\text { multicenter } \\
\text { trial }\end{array}$ & $\begin{array}{l}18 \text { years or } \\
\text { older }\end{array}$ & \begin{tabular}{|l}
$50.8 \%$ in FVP \\
group/ 42.5\% \\
in Arbidol \\
group
\end{tabular} & $\begin{array}{l}\mathrm{FPV}(1600 \\
\mathrm{mg}^{\star 2} 2 / \text { first day } \\
\text { followed by } 600 \\
\mathrm{mg}^{\star} 2 / \text { day) for } \\
10 \text { days/ } 116\end{array}$ & $\begin{array}{l}\text { Umifenovir } \\
\text { (Arbidol) }(200 \\
\left.\mathrm{mg}^{*} 3 / \text { day }\right) / 120\end{array}$ & Day 10 \\
\hline $\begin{array}{l}\text { Dabbous } \\
\text { et al./2020 }\end{array}$ & NCT04349241 & Preprint & $\begin{array}{l}\text { Research } \\
\text { Square }\end{array}$ & Egypt & $\begin{array}{l}\text { Computer } \\
\text { based } \\
\text { randomized } \\
\text { controlled } \\
\text { interven- } \\
\text { tional clinical } \\
\text { trial phase } 3\end{array}$ & $18-80$ & $\begin{array}{l}50 \% \text { in each } \\
\text { groups }\end{array}$ & $\begin{array}{l}\text { FPV } 3200 \mathrm{mg} \text { at } \\
\text { dayl followed } \\
\text { by } 600 \mathrm{mg} \\
\text { twice }(\text { day2- } \\
\text { day10)/50 }\end{array}$ & \begin{tabular}{|l} 
HCQ $800 \mathrm{mg}$ at \\
dayl followed \\
by $200 \mathrm{mg}$ twice \\
(day2-10) and \\
oral oseltamivir \\
$75 \mathrm{mg} / 12 \mathrm{~h} /$ day \\
for 10 days/ 50
\end{tabular} & Day 30 \\
\hline $\begin{array}{l}\text { Doi } \\
\text { et al./2020 }\end{array}$ & jRCTs041190120 & In press & $\begin{array}{l}\text { Antimicrobial } \\
\text { Agents and } \\
\text { Chemo- } \\
\text { therapy }\end{array}$ & Japan & $\begin{array}{l}\text { Prospective, } \\
\text { randomized, } \\
\text { open -label, } \\
\text { multicenter } \\
\text { trial }\end{array}$ & $\begin{array}{l}16 \text { years or } \\
\text { older }\end{array}$ & \begin{tabular}{|l}
$52.3 \%$ in \\
early group, \\
$705 . \%$ in late \\
group
\end{tabular} & \begin{tabular}{|l} 
Early FPV: \\
Favipiravir was \\
dosed at 1800 \\
mg orally at \\
least four hours \\
apart on the first \\
day, followed \\
by 800 \\
278 mg orally \\
twice a day, for \\
a total of up to \\
19 doses over 10 \\
days/ 36
\end{tabular} & $\begin{array}{l}\text { Late FVP: Favip- } \\
\text { iravir was dosed } \\
\text { at } 1800 \text { mg orally } \\
\text { at least four hours } \\
\text { apart on the first } \\
\text { day, followed by } \\
800 / 33\end{array}$ & Day 28 \\
\hline \multirow{2}{*}{$\begin{array}{l}\text { Ivashchenko } \\
\text { et al./2020 }\end{array}$} & \multirow{2}{*}{ NCT04434248 } & \multirow{2}{*}{ In press } & \multirow{2}{*}{$\begin{array}{l}\text { Clinical Infec- } \\
\text { tious Disease }\end{array}$} & \multirow{2}{*}{ Russia } & \multirow{2}{*}{$\begin{array}{l}\text { Adaptive, } \\
\text { multicenter, } \\
\text { open label, } \\
\text { randomized, } \\
\text { phase } 2 \text { and } 3 \\
\text { clinical trial }\end{array}$} & \multirow{2}{*}{$\begin{array}{l}18 \text { years or } \\
\text { older }\end{array}$} & \multirow{2}{*}{ NR } & $\begin{array}{l}\text { AVIFAVIR } 1600 \\
\text { mg BID on Day } \\
1 \text { followed by } \\
600 \mathrm{mg} \text { BID } \\
\text { on Days } 2-14 \\
(1600 / 600 \\
\mathrm{mg}) / 20\end{array}$ & \multirow{2}{*}{$\begin{array}{l}\text { Standard of } \\
\text { care of Russian } \\
\text { guidelines for } \\
\text { treatment of } \\
\text { COVID-19/20 }\end{array}$} & \multirow{2}{*}{ Day 29} \\
\hline & & & & & & & & \begin{tabular}{|l|} 
AVIFAVIR 1800 \\
mg BID on Day \\
1 followed by \\
$800 \mathrm{mg}$ BID \\
on Days $2-14$ \\
$(1800 / 800$ \\
$\mathrm{mg}) / 20$
\end{tabular} & & \\
\hline $\begin{array}{l}\text { Khamis } \\
\text { et al./2021 }\end{array}$ & NCT04385095 & $\begin{array}{l}\text { Published } \\
\text { online }\end{array}$ & \begin{tabular}{|l|} 
International \\
Journal of \\
Infectious \\
Diseases
\end{tabular} & Oman & $\begin{array}{l}\text { Open label } \\
\text { randomized } \\
\text { controlled } \\
\text { study }\end{array}$ & $18-75$ & $\begin{array}{l}64 \% \text { in FVP } \\
\text { group } / 53 \% \text { in } \\
\text { SOC group }\end{array}$ & \begin{tabular}{|l} 
FPV $1600 \mathrm{mg}$ \\
on day 1 fol- \\
lowed by 600 \\
$\mathrm{mg}$ twice a day \\
for a maximum \\
of 10 days, and \\
interferon beta- \\
$1 \mathrm{~b}$ at a dose of 8 \\
million IU ( 0.25 \\
$\mathrm{mg})$ twice a day \\
was given for \\
5 days through \\
a vibrating \\
mesh aerogen \\
nebulizer/ 44
\end{tabular} & \begin{tabular}{|l|} 
Standard of \\
care of Oman \\
guidelines for \\
treatment of \\
COVID-19: HCQ \\
400 mg twice \\
per day on day \\
1 , then $200 \mathrm{mg}$ \\
twice per day for \\
7 days $/ 45$
\end{tabular} & Day 14 \\
\hline
\end{tabular}




\begin{tabular}{|c|c|c|c|c|c|c|c|c|c|c|}
\hline Authors/year & $\begin{array}{l}\text { ClinicalTrials } \\
\text { identifier }\end{array}$ & $\begin{array}{l}\text { Publication } \\
\text { status }^{\mathrm{a}}\end{array}$ & Journal & Country & Study design & Age & Male & $\begin{array}{l}\text { Intervention/ } \\
\text { sample size }\end{array}$ & $\begin{array}{l}\text { Comparison/ } \\
\text { sample size }\end{array}$ & Follow up \\
\hline \multirow[t]{2}{*}{$\begin{array}{l}\text { Lou } \\
\text { et al./2020 }\end{array}$} & \multirow[t]{2}{*}{ ChiCTR2000029544 } & \multirow[t]{2}{*}{ Published } & \multirow{2}{*}{$\begin{array}{l}\text { European } \\
\text { Journal of } \\
\text { Pharmaceuti- } \\
\text { cal Sciences }\end{array}$} & \multirow[t]{2}{*}{ China } & \multirow{2}{*}{$\begin{array}{l}\text { Exploratory } \\
\text { single center, } \\
\text { open-label, } \\
\text { randomized, } \\
\text { controlled } \\
\text { trial }\end{array}$} & \multirow{2}{*}{$\begin{array}{l}\text { Mean: } 58, \\
53.5 \text { and } \\
46.6 \text { for FAV, } \\
\text { Baloxavir } \\
\text { and control } \\
\text { group }\end{array}$} & \multirow{2}{*}{$\begin{array}{l}77 \% \text { in FVP } \\
\text { group/ } 70 \% \\
\text { in other } \\
\text { groups }\end{array}$} & \multirow{2}{*}{$\begin{array}{l}\text { FAV group: } \\
1600 \mathrm{mg} \text { or } \\
2200 \mathrm{mg} \text { orally, } \\
\text { followed by } 600 \\
\text { mg each time, } \\
\text { three times a } \\
\text { day, and the } \\
\text { duration of } \\
\text { administration } \\
\text { was not more } \\
\text { than } 14 \text { days } / 9\end{array}$} & $\begin{array}{l}\text { Baloxavirmar- } \\
\text { boxil group: } 80 \\
\text { mg once a day } \\
\text { orally on Day } 1 \\
\text { and Day 4; for } \\
\text { patients who are } \\
\text { still positive in } \\
\text { virological test, } \\
\text { they can be given } \\
\text { again on Day } \\
7 / 10\end{array}$ & \multirow[t]{2}{*}{ Day 14} \\
\hline & & & & & & & & & $\begin{array}{l}\text { Control group: } \\
\text { LPV/RTV (400 } \\
\text { mg/100 mg, bid, } \\
\text { po.) or darunavir/ } \\
\text { cobicistat ( } 800 \\
\text { mg/150 mg, qd, } \\
\text { po.) and arbidol } \\
\text { (200 mg, tid, } \\
\text { po.)/ } 10\end{array}$ & \\
\hline $\begin{array}{l}\text { Udwadia } \\
\text { et al./2020 }\end{array}$ & CTRI/2020/05/025114 & Published & $\begin{array}{l}\text { International } \\
\text { Journal of } \\
\text { Infectious } \\
\text { Diseases }\end{array}$ & India & $\begin{array}{l}\text { Randomized, } \\
\text { open-label, } \\
\text { parallel-arm, } \\
\text { multicenter, } \\
\text { phase } 3 \text { study }\end{array}$ & $18-75$ & $\begin{array}{l}70.8 \% \text { in FVP } \\
\text { group/76\% in } \\
\text { control group }\end{array}$ & $\begin{array}{l}\text { Oral Favipiravir } \\
\text { (1800 mg } \\
\text { BID loading } \\
\text { dose on day } 1 ; \\
800 \text { mg BID } \\
\text { maintenance } \\
\text { dose thereafter) } \\
\text { plus standard } \\
\text { supportive } \\
\text { care for up to a } \\
\text { maximum of } 14 \\
\text { days/70 }\end{array}$ & $\begin{array}{l}\text { Standard sup- } \\
\text { portive care alone } \\
\text { that included } \\
\text { antipyretics, } \\
\text { cough suppres- } \\
\text { sants, antibiotics, } \\
\text { and vitamins/68 }\end{array}$ & Day 14 \\
\hline \multirow[b]{2}{*}{$\begin{array}{l}\text { Zhao } \\
\text { et al./2021 }\end{array}$} & \multirow[b]{2}{*}{$\begin{array}{l}\text { ChiCTR2000030894 } \\
\text { and NCT04310228 }\end{array}$} & \multirow[b]{2}{*}{$\begin{array}{l}\text { Published } \\
\text { online }\end{array}$} & \multirow[b]{2}{*}{$\begin{array}{l}\text { Biomedicine } \\
\text { \& Pharmaco- } \\
\text { therapy }\end{array}$} & \multirow[b]{2}{*}{ China } & \multirow[b]{2}{*}{$\begin{array}{l}\text { Multicenter, } \\
\text { randomized } \\
\text { trial }\end{array}$} & \multirow[b]{2}{*}{$\begin{array}{l}18 \text { years or } \\
\text { older }\end{array}$} & \multirow[b]{2}{*}{$\begin{array}{l}71.4 \% \text { in FVP } \\
\text { group/60\% in } \\
\text { Tocilizumab } \\
\text { group }\end{array}$} & \multirow{2}{*}{$\begin{array}{l}\text { FAV group: } \\
1600 \mathrm{mg} \text {, twice } \\
\text { a day on the } \\
\text { first day, and } \\
600 \mathrm{mg} \text {, twice } \\
\text { a day from the } \\
\text { second day to } \\
\text { the seventh day, } \\
\text { orally/7 }\end{array}$} & $\begin{array}{l}\text { Combina- } \\
\text { tion group } \\
\text { (FAV + tocili- } \\
\text { zumab)/14 }\end{array}$ & \\
\hline & & & & & & & & & $\begin{array}{l}\text { Tocilizumab } \\
\text { group: first dose } \\
\text { was } 4-8 \mathrm{mg} / \mathrm{kg} \\
\text { (recommended } \\
400 \mathrm{mg} \text { ) and } \\
\text { added to } 100 \\
\text { mL } 0.9 \% \text { normal } \\
\text { saline } / 5\end{array}$ & \\
\hline
\end{tabular}

Table 1. summary characteristics of the included studies. NR not reported, FPV Favipiravir, $L P V$ Lopinavir, $R T V$ Ritonavir, HCQ hydroxychloroquine. ${ }^{a}$ The status of manuscript in time of screening.

Risk of bias in individual studies. Eight (88.8\%) studies described the random sequence generation. Six studies $(66.6 \%)$ described the allocation concealment in an acceptable manner. None of the studies reported acceptable blinding for participants and personnel. Only one study (11.1\%) reported blinding of outcome assessment. The risk of bias summary and risk of bias graph is reported in Supplementary file 2 and 3.

The results of the meta-analysis. Clinical improvement. Among the included studies, six studies assessed clinical improvement during 14 days after hospitalization, and five studies were assessed during seven days after hospitalization. The results of the meta-analysis revealed a significant clinical improvement in the Favipiravir group versus the control group during seven days after hospitalization (RR =1.24, 95\% CI: 1.09-1.41; $\left.\mathrm{P}=0.001, \mathrm{I}^{2}=0.0 \%, \mathrm{P}=0.939\right)$. On the other hand, in 14 days after hospitalization, clinical improvement was $10 \%$ higher in the Favipiravir group, but this finding not statistically significant $(R R=1.10,95 \%$ CI: $0.97-1.25$; $\mathrm{P}=0.108, \mathrm{I}^{2}=34.5 \%, \mathrm{P}=0.177$ ) (Fig. 2).

Viral clearance. The result of meta-analysis show that, viral clearance was more in 14 days after hospitalization in Favipiravir group than control group, but this finding marginally not significant $(\mathrm{RR}=1.11,95 \% \mathrm{CI}$ : $\left.0.98-1.25 ; \mathrm{P}=0.094, \mathrm{I}^{2}=42.9 \%, \mathrm{P}=0.112\right)$. Viral clearance in 7 and 10 days after hospitalization not statistically different between two groups $\left(\mathrm{RR}=1.07,95 \% \mathrm{CI}\right.$ : $0.83-1.39 ; \mathrm{P}=0.580, \mathrm{I}^{2}=62.1 \%, \mathrm{P}=0.022$ for 7 days and $\mathrm{RR}=1.02,95 \%$ CI: $0.92-1.13 ; \mathrm{P}=0.648, \mathrm{I}^{2}=0.0 \%, \mathrm{P}=0.846$ for 10 days) (Fig. 3 ).

Requiring supplemental oxygen therapy. Based on the meta-analysis, requiring supplemental oxygen therapy in the Favipiravir group was $7 \%$ less than the control group, but this finding not statistically significant $\left(\mathrm{RR}=0.93,95 \%\right.$ CI: 0.67-1.28; $\left.\mathrm{P}=0.664, \mathrm{I}^{2}=0.0 \%, \mathrm{P}=0.950\right)$ (Fig. 4). 


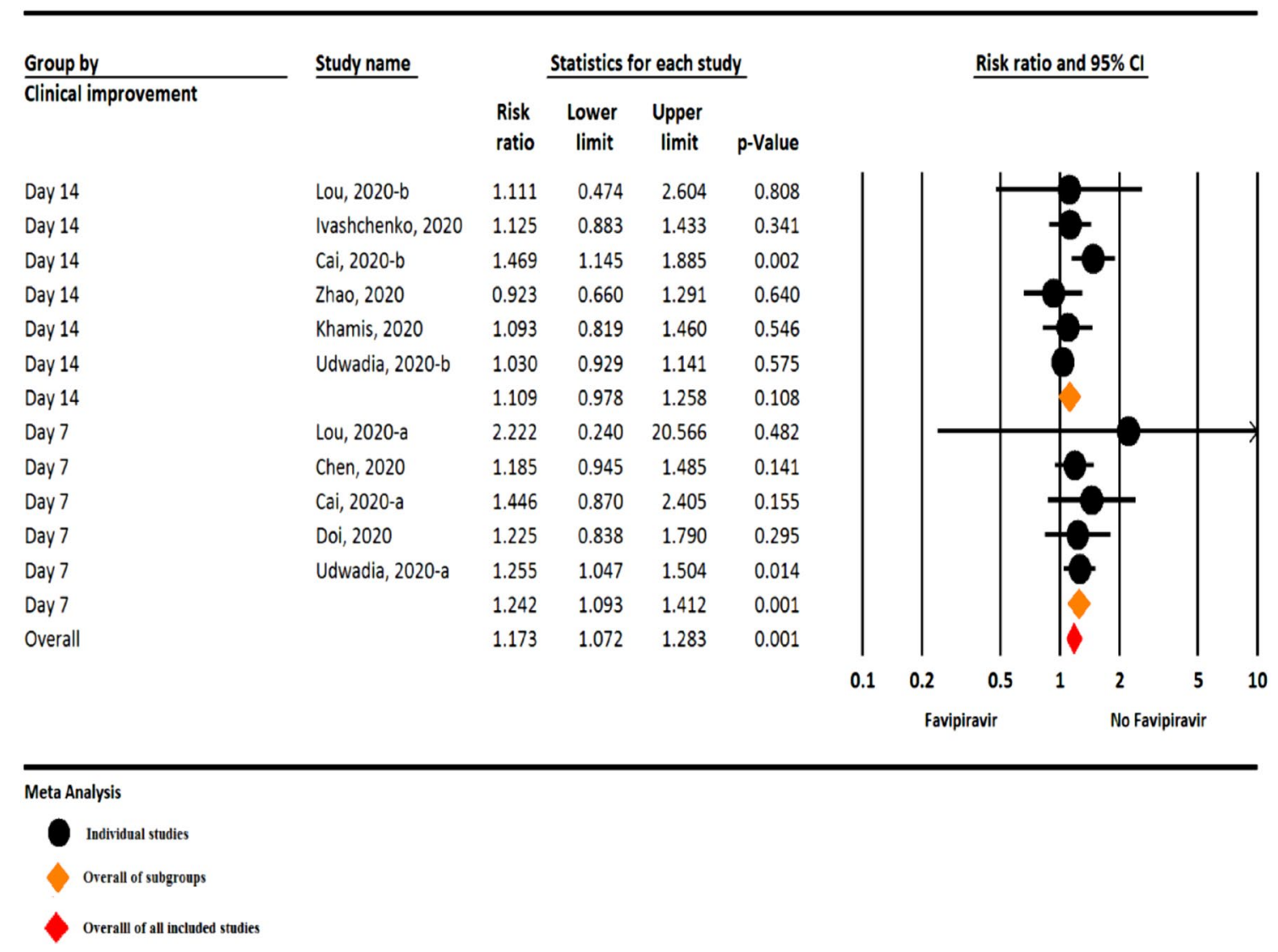

Figure 2. The meta-analysis of clinical improvement of Favipiravir on COVID-19 patients (black circle: individual studies; orange diamond: overall of subgroups; red diamond: overall of all included studies).

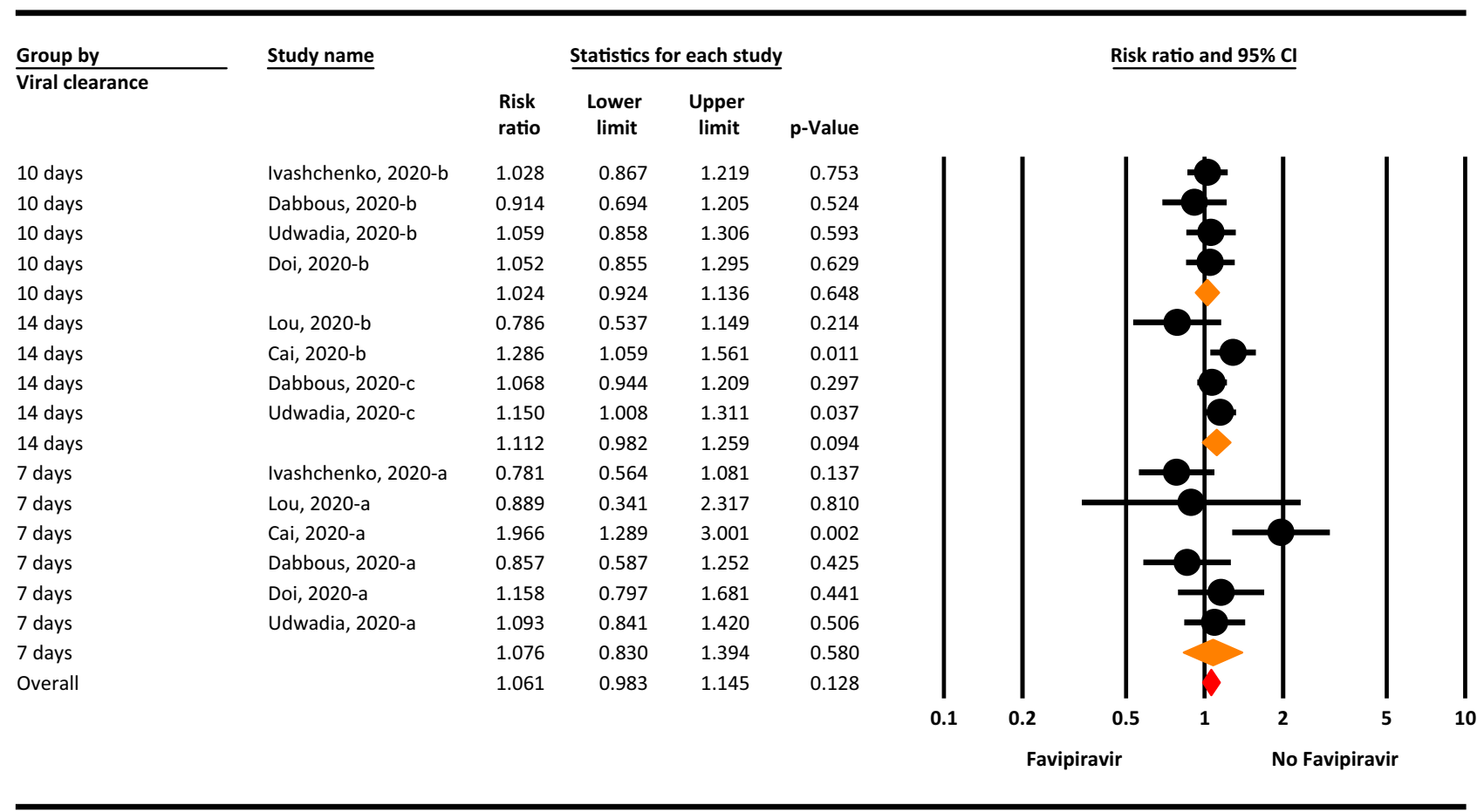

Figure 3. The meta-analysis of viral clearance of Favipiravir on COVID-19 patients (orange diamond: summery of sub groups; red diamond: summery of total). 
Study name

\begin{tabular}{|c|c|c|c|c|c|c|c|c|c|c|}
\hline & $\begin{array}{l}\text { Risk } \\
\text { ratio }\end{array}$ & $\begin{array}{l}\text { Lower } \\
\text { limit }\end{array}$ & $\begin{array}{l}\text { Upper } \\
\text { limit }\end{array}$ & p-Value & & & & & & \\
\hline Lou, 2020 & 0.833 & 0.252 & 2.755 & 0.765 & & & & & & \\
\hline Udwadia, 2020 & 1.000 & 0.369 & 2.712 & 1.000 & & & & & & \\
\hline Ivashchenko, 2020 & 1.000 & 0.395 & 2.534 & 1.000 & & & & & & \\
\hline Chen, 2020 & 0.805 & 0.483 & 1.340 & 0.403 & & & & & & \\
\hline \multirow[t]{4}{*}{ Zhao, 2020} & 1.111 & 0.604 & 2.045 & 0.735 & & & & & & \\
\hline & 0.930 & 0.671 & 1.289 & 0.664 & & & & & & \\
\hline & & & & & 0.1 & 0.2 & 0.5 & & 2 & 5 \\
\hline & & & & & \multicolumn{4}{|c|}{ Favipiravir } & & \\
\hline
\end{tabular}

Meta Analysis

Figure 4. The meta-analysis of requiring supplemental oxygen therapy of Favipiravir on COVID-19 patients (red diamond: summery of total).
Study name

$\begin{array}{lrrrr} & \begin{array}{c}\text { Risk } \\ \text { ratio }\end{array} & \begin{array}{r}\text { Lower } \\ \text { limit }\end{array} & \begin{array}{c}\text { Upper } \\ \text { limit }\end{array} & \text { p-Value } \\ \text { Ivashchenko, 2020 } & 1.500 & 0.636 & 3.538 & 0.354 \\ \text { Cai, 2020 } & 0.206 & 0.079 & 0.537 & 0.001 \\ \text { Chen, 2020 } & 1.367 & 0.899 & 2.079 & 0.144 \\ \text { Zhao, 2020 } & 0.444 & 0.129 & 1.528 & 0.198 \\ \text { Lou, 2020 } & 0.741 & 0.305 & 1.801 & 0.508 \\ \text { Khamis, 2020 } & 0.205 & 0.086 & 0.486 & 0.000 \\ \text { Udwadia, 2020 } & 4.500 & 1.973 & 10.265 & 0.000 \\ & 0.772 & 0.349 & 1.709 & 0.524\end{array}$

$\underline{\text { Risk ratio and } 95 \% \mathrm{Cl}}$

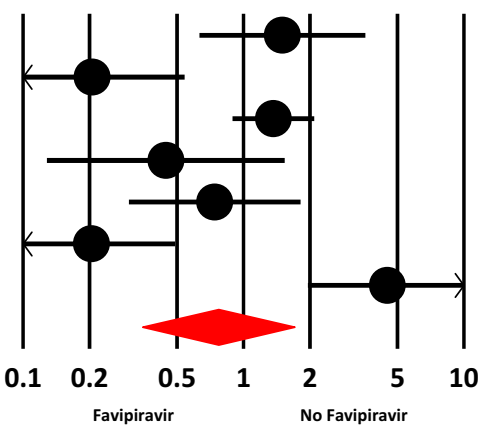

Meta Analysis

Figure 5. The meta-analysis of adverse events of Favipiravir on COVID-19 patients (red diamond: summery of total).

Adverse events. Meta-analysis comparing adverse events between the Favipiravir and the control groups showed lesser odds for adverse effects in the Favipiravir arm but of no statistical significance $(\mathrm{RR}=0.77,95 \% \mathrm{CI}$ : 0.34-1.70; $\mathrm{P}=0.524, \mathrm{I}^{2}=85.4 \%, \mathrm{P}<0.001$ ) (Fig. 5).

Almost all adverse events of Favipiravir were mild to moderate and in an equal or lower rate compared to the control groups. The most prevalent adverse events included nausea, vomiting, diarrhea, chest pain as well as increase in serum liver transaminase and uric acid levels.

Transferred to ICU. Based on meta-analysis, transferred to ICU not statistically different between two groups ( $\mathrm{RR}=1.13,95 \% \mathrm{CI}$ : $\left.0.49-2.59 ; \mathrm{P}=0.759, \mathrm{I}^{2}=0.0 \%, \mathrm{P}=0.525\right)$ (Fig. 6).

Mortality. Based on the meta-analysis, the mortality rate in the Favipiravir group was approximately $30 \%$ less than the control group, but this finding not statistically significant $(\mathrm{RR}=0.70,95 \% \mathrm{CI}: 0.26-1.28 ; \mathrm{P}=0.664$, $\mathrm{I}^{2}=0.0 \%, \mathrm{P}=0.950$ ) (Fig. 7).

Sensitivity analysis. The results of sensitivity analysis did not show any difference in all outcomes and confirmed the previous results.

Publication bias. Publication bias was not observed among the included studies according to the results of the Egger $(\mathrm{P}=0.944)$ and Begg test $(\mathrm{P}=0.956)$. 
Study name

$\begin{array}{lcrrr} & \begin{array}{c}\text { Risk } \\ \text { ratio }\end{array} & \begin{array}{r}\text { Lower } \\ \text { limit }\end{array} & \begin{array}{c}\text { Upper } \\ \text { limit }\end{array} & \text { p-Value } \\ \text { Khamis, 2020 } & 1.023 & 0.421 & 2.484 & 0.960 \\ \text { Lou, 2020 } & 2.222 & 0.240 & 20.566 & 0.482 \\ & 1.138 & 0.499 & 2.594 & 0.759\end{array}$

Risk ratio and $95 \% \mathrm{Cl}$

Statistics for each study

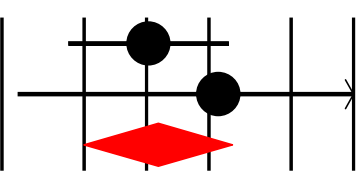

$\begin{array}{lllllll}0.1 & 0.2 & 0.5 & 1 & 2 & 5 & 10\end{array}$

Favipiravir No Favipiravir

Meta Analysis

Figure 6. The meta-analysis of transferred to ICU of Favipiravir on COVID-19 patients (red diamond: summery of total).

Study name

$\begin{array}{lcccr} & \begin{array}{c}\text { Risk } \\ \text { ratio }\end{array} & \begin{array}{c}\text { Lower } \\ \text { limit }\end{array} & \begin{array}{c}\text { Upper } \\ \text { limit }\end{array} & \text { p-Value } \\ \text { Dabbous, 2020 } & 0.333 & 0.014 & 7.991 & 0.498 \\ \text { Khamis, 2020 } & 0.852 & 0.280 & 2.590 & 0.778 \\ \text { Udwadia, 2020 } & 0.333 & 0.014 & 8.054 & 0.499 \\ & 0.709 & 0.262 & 1.920 & 0.499\end{array}$

Statistics for each study
Risk ratio and $95 \% \mathrm{Cl}$

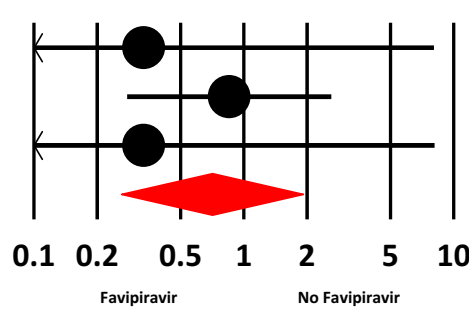

Meta Analysis

Figure 7. The meta-analysis of mortality of Favipiravir on COVID-19 patients (red diamond: summery of total).

\section{Discussion}

The COVID-19 causing SARS-CoV-2, an acute respiratory disease, is spreading rapidly and has led to a pandemic with devastating effects within a few months of November $2019^{38}$. The number of infected cases, as well as the mortality rate associated with the virus, has astronomically raised around the world. The main challenge of COVID-19 is the lack of approved pharmacotherapy and vaccination, as well as the absence of evidence for reliable treatment options ${ }^{38}$. Although various agents are undergoing clinical trials, the urgency of the situation has made scientists repurpose the antiviral agents.

Favipiravir, as a ribonucleotide analog and selective inhibitor of the viral RNA polymerase enzyme, can cause widespread antiviral activity against RNA-carrying viruses, thereby preventing replication and transcription of the viral genome ${ }^{39}$. It has been approved for the treatment of new influenza viruses in Japan and China. It has also been shown to be effective against Ebola and RNA viruses caused by viral hemorrhagic fever ${ }^{31}$.

However, none of the society and organizational guidelines (IDSA guidelines, World Health Organization guidelines, National Institutes of Health guidelines) recommend using Favipiravir in the management of COVID19, given the varying results of existing clinical trials data ${ }^{36}$. Moreover, this drug revealed controversial results in different clinical trials conducted on COVID-19. Therefore, we decided to investigate the safety and efficacy of Favipiravir in the treatment of COVID-19. Our meta-analysis was carried out on nine eligible studies with 827 patients.

The obtained results demonstrated the clinical improvement after seven and 14 days of hospitalization was more remarkable in patients taking Favipiravir than those receiving other drugs. Another meta-analysis conducted by Shrestha et al. demonstrated that clinical improvement was observed on both the seventh and 14th day of treatment ${ }^{40}$. Udwadia et al. reported the time of clinical improvement was significantly faster in patients in the Favipiravir group than those who are not ${ }^{36}$.

The viral clearance after 14 days of hospitalization among patients taking Favipiravir was more than those taking other drugs. However, this difference was not statistically significant after seven and ten days, which could be related to inappropriate dose and duration of treatment with Favipiravir ${ }^{35}$. In another meta-analysis by 
Shrestha et al., it was stated that viral clearance on seventh and 14th was not significant between the Favipiravir and control groups ${ }^{40}$.

This difference between the results of our analysis and the mentioned meta-analysis might be due to an insufficient number of studies and a small sample size in the Shrestha et al. meta-analysis. Adolfo Pérez-García et al. reported that a randomized study on 80 patients with mild COVID-19 showed Favipiravir group reduced virus clearance time by $50 \%$ compared to Lopinavir/Ritonavir group ${ }^{39}$.

Our study showed requiring supplemental oxygen therapy among patients taking Favipiravir was less than those taking control drugs. Dhan Bahadur Shrestha et al. also showed that patients receiving Favipiravir had less need for oxygen and non-invasive mechanical ventilation ${ }^{40}$.

The results of the present study showed that the groups treated with Favipiravir had a lower chance of side effects compared to the control groups. This finding is consistent with the meta-analysis carried out by Shrestha et al. $^{40}$. Khamis et al. also found intervention with Favipiravir had no significant side effects, including hyperuricemia, abnormalities in liver enzymes, or QTc prolongation ${ }^{34}$.

Erdem et al. found that side effects occurred in $13 \%$ of patients during treatment with Favipiravir. The most common side effects were elevation of liver enzymes, total bilirubin, and uric acid, as well as gastrointestinal disorders. This trial consists of five patients, and All five experienced mild to moderate rise in liver enzymes, three of them nausea, and one of them neutropenia. All side effects were self-limited. There was no association between underlying disease and serious side effects, and no patients stopped Favipiravir due to side effects ${ }^{38}$. Victoria Pilkington et al. demonstrated that patients who took Favipiravir had no serious side effects. However, an increase in serum uric acid remains a concern, and the analysis of studies showed some evidence of a dosedependent increase in this biochemical parameter. Other complications, including teratogenic potential and QTc prolongation, have not been sufficiently studied ${ }^{41}$. Denis Malvy et al. also reported that Favipiravir is well tolerated and safe in short-term administration. However, more evidence is necessary to conclude long-term safety $^{42}$. Udwadia et al. reported most of the side effects were mild to moderate, and the most common side effects were an asymptomatic transient rise in serum uric acid and liver enzymes. On the other hand, gastrointestinal disorders were minimal ${ }^{36}$.

Totally, intervention with Favipiravir exerted minor tolerable side effects, including nausea, vomiting, diarrhea, and elevated serum transaminases. There were no serious life-threatening side effects after treatments with Favipiravir. Possible side effects could not be attributed to the only consumption of Favipiravir. Patients in the Favipiravir groups received other drugs in all three trials ${ }^{40}$.

Our analysis showed the need for admission in ICU is not statistically significant between the Favipiravir groups and control groups. Khamis et al. also revealed there was no significant difference between Favipiravir and hydroxychloroquine group in the case of transfer to $\mathrm{ICU}^{34}$. Additionally, Yan Lou et al. found only two of the 22 patients in Favipiravir and one patient in baloxavir marboxil group transferred to the ICU within seven days of starting intervention ${ }^{35}$.

Based on the results of the analysis, there has been a decrease in all-cause mortality in patients who took Favipiravir compared to those who took control of drugs. In a study carried out by Dabbous, one patient in the hydroxychloroquine group expired. However, no death was reported in the Favipiravir group ${ }^{31}$.

Considering the importance of treating patients with COVID-19, further studies on the role of Favipiravir in the management of COVID-19 patients are recommended. Despite the limitation, the present study provided the information needed for treating COVID-19, suggesting that Favipiravir is associated with significant clinical and laboratory improvement in most patients and it is a safe drug with no serious side effects ${ }^{38}$.

There is some evidence to support the safety and tolerability of Favipiravir in short-term administration. However, more evidence is necessary to evaluate the exact long-term effects of this intervention. Due to limited evidence and other specific safety concerns, caution should be considered in the widespread use of Favipiravir against the COVI D-19 epidemic ${ }^{43}$.

Limitations. There are some limitations to the included studies. First, the sample size is low in each study. Second, due to multiple drug pharmacotherapy of patients with COVID-19 in the most included study, there was, therefore, a risk of influencing the efficacy and also the safety of intervention with Favipiravir. Third, the dosage and duration of intervention with Favipiravir are different among the included studies. Fourth, it is difficult to determine the clinical improvement found in patients treated with Favipiravir from different disease severity, ages, and medical conditions in the different studies.

\section{Conclusion}

Overall, Favipiravir possibly exerted no significant beneficial effect in the term of mortality in the general group of patients with mild to moderate COVID-19. We should consider that perhaps the use of antivirals once the patient has symptoms is too late and this would explain their low efficacy in the clinical setting. There upon, more clinical trials with a larger sample size are necessary to evaluate the exact efficacy and safety of this intervention.

Received: 10 January 2021; Accepted: 13 May 2021

Published online: 26 May 2021

\section{References}

1. Arab-Zozani, M. \& Hassanipour, S. Features and limitations of LitCovid hub for quick access to literature about COVID-19. Balkan. Med. J. 37, 231. https://doi.org/10.4274/balkanmedj.galenos.2020.2020.4.67 (2020). 
2. Fact Sheet for "the Biopharmaceutical Industry Leading the Way in Developing New Vaccines and Treatments for COVID-19" Available at: https://phrma.org/Coronavirus (2021).

3. Arab-Zozani, M., Hassanipour, S. \& Ghoddoosi-Nejad, D. Favipiravir for treating patients with novel coronavirus (COVID-19): Protocol for a systematic review and meta-analysis of randomised clinical trials. BMJ. Open. 10, e039730. https://doi.org/10.1136/ bmjopen-2020-039730 (2020).

4. Food, U. \& Administration, D. Fact Sheet for Health Care Providers Emergency Use Authorization (EUA) of Veklury (remdesivir). (2020).

5. Rubin, D., Chan-Tack, K., Farley, J., Sherwat, A. FDA approval of remdesivir-a step in the right direction. New Engl. J. Med. 383, 2598-2600. https://doi.org/10.1056/NEJMp2032369 (2020).

6. Hippensteele, A. FDA Issues Emergency Use Authorization for Convalescent Plasma Treatment for Patients with COVID-19. Avaialble at: https://www.pharmacytimes.com/view/fda-issues-emergency-use-authorization-for-convalescent-plasma-treatment-for-patie nts-with-covid-19?sp_url=coronavirus (2020).

7. Fact sheet for healthcare providers emergency use authorization (EUA) ofbamlanivimab. United States Food and Drug Administration. https://www.fda.gov/media/143603/download. (2020). Accessed 10 Nov 2020.

8. FDA. Fact sheet for healthcare providers emergency use authorization (EUA) of baricitinib. fda.gov. https://www.fda.gov/media/ 143823/download. (2020). Accessed 19 Nov 2020.

9. FDA. Fact sheet for healthcare providers emergency use authorization (EUA) of casirivimab and imdevimab. United States Food and Drug Administration. https://www.fda.gov/media/143892/download. (2020). Accessed 21 Nov 2020

10. Fink, D. Considerations for FDA licensure vs. emergency use authorization of COVID-19 vaccines. (2020).

11. The Milken Institute. COVID-19 Treatment and Vaccine Tracker. https://covid-19tracker.milkeninstitute.org/. Accessed 14 May 2021.

12. Konstantinidou, S. K. \& Papanastasiou, I. P. Repurposing current therapeutic regimens against SARS-CoV-2 (Review). Exp. Ther. Med. 20, 1845-1855. https://doi.org/10.3892/etm.2020.8905 (2020).

13. Furuta, Y. et al. In vitro and in vivo activities of anti-influenza virus compound T-705. Antimicrob. Agents Chemother. 46, 977-981 (2002).

14. Agrawal, U., Raju, R. \& Udwadia, Z. F. Favipiravir: A new and emerging antiviral option in COVID-19. Med. J. Armed Forces India 76, 370-376. https://doi.org/10.1016/j.mjafi.2020.08.004 (2020).

15. Allen, C. N. S., Arjona, S. P., Santerre, M. \& Sawaya, B. E. Potential use of RNA-dependent RNA polymerase (RdRp) inhibitors against SARS-CoV2 infection. All Life 13, 608-614. https://doi.org/10.1080/26895293.2020.1835741 (2020).

16. Clinicaltrials.gov. Search of: Favipiravir j Covid19-listresults-clinicaltrials.gov (online) https://clinicaltrials.gov/ct2/results?cond= Covid19\&term $=$ Favipiravir\&cntry $=\&$ state $=\& c i t y=\&$ dist. $(2020)$. Accessed 12 Oct 2020.

17. Karlsen, A. P. H. et al. A systematic review of trial registry entries for randomized clinical trials investigating COVID-19 medical prevention and treatment. PLoS ONE 15, e0237903. https://doi.org/10.1371/journal.pone.0237903 (2020).

18. Liu, W. et al. Efficacy and safety of antiviral treatment for COVID-19 from evidence in studies of SARS-CoV-2 and other acute viral infections: A systematic review and meta-analysis. CMAJ Can. Med. Assoc. J. (J. l’Assoc. Med. Can.) 192, E734-E744. https:// doi.org/10.1503/cmaj.200647 (2020).

19. Misra, S., Nath, M., Hadda, V. \& Vibha, D. Efficacy of various treatment modalities for nCOV-2019: A systematic review and meta-analysis. Eur. J. Clin. Invest. 50, https://doi.org/10.1111/eci.13383 (2020).

20. Nasir, M. et al. Systematic review on repurposing use of Favipiravir against SARS-CoV-2. Mymensingh Med. J. (MMJ) 29, 747-754 (2020).

21. Siemieniuk, R. A. C. et al. Drug treatments for covid-19: Living systematic review and network meta-analysis. BMJ 370, https:// doi.org/10.1136/bmj.m2980 (2020).

22. Moher, D., Liberati, A., Tetzlaff, J. \& Altman, D. G. Preferred reporting items for systematic reviews and meta-analyses: The PRISMA statement. Ann. Intern. Med. 151, 264-269 (2009).

23. WHO. Clinical Management of COVID-19: Interim Guidance. https://www.who.int/publications/i/item/clinical-management-ofcovid-19 (2020).

24. Chen, Q., Allot, A. \& Lu, Z. Keep up with the latest coronavirus research. Nature 579, 193 (2020).

25. Arab-Zozani, M., Pezeshki, M. Z., Khodayari-Zarnaq, R. \& Janati, A. Medical overuse in the Iranian healthcare system: A systematic review protocol. BMJ Open 8, e020355. https://doi.org/10.1136/bmjopen-2017-020355 (2018).

26. Morteza, A.-Z., Soheil, H. \& Djavad, G.-N. Favipiravir for treating novcl coronavirus (COVID-19) patients: Protocol for a systematic review and meta-analysis of Controlled trials. Res. Square https://doi.org/10.21203/rs.3.rs-23106/v1 (2021).

27. Hassanipour, S. et al. Comparison of artificial neural network and logistic regression models for prediction of outcomes in trauma patients: A systematic review and meta-analysis. Injury 50, 244-250 (2019).

28. Huedo-Medina, T. B., Sánchez-Meca, J., Marín-Martínez, F. \& Botella, J. Assessing heterogeneity in meta-analysis: Q statistic or $\mathrm{I}^{2}$ index?. Psychol. Methods 11, 193 (2006).

29. Cai, Q. et al. Experimental treatment with Favipiravir for COVID-19: An open-label control study. Engineering (Beijing, China) 6, 1192-1198. https://doi.org/10.1016/j.eng.2020.03.007 (2020).

30. Chen, P. J., Chao, C. M. \& Lai, C. C. Clinical efficacy and safety of Favipiravir in the treatment of COVID-19 patients. J. Infect. https://doi.org/10.1016/j.jinf.2020.12.005 (2020).

31. Dabbous, H. M., El-Sayed, M. H., El Assal, G., Elghazaly, H., Ebeid, F. F., Sherief, A. F. et al. Safety and efficacy of favipiravir versus hydroxychloroquine in management of COVID-19: A randomised controlled trial. Sci. Rep. 11, 1-7 (2021).

32. Doi, Y. et al. A prospective, randomized, open-label trial of early versus late favipiravir therapy in hospitalized patients with COVID-19. Antimicrob. Agents Chemother. 64, https://doi.org/10.1128/aac.01897-20 (2020).

33. Ivashchenko, A. A. et al. AVIFAVIR for treatment of patients with moderate COVID-19: Interim results of a phase II/III multicenter randomized clinical trial. Clin. Infect. Dis. https://doi.org/10.1093/cid/ciaal176 (2020).

34. Khamis, F. et al. Randomized controlled open label trial on the use of Favipiravir combined with inhaled interferon beta-1b in hospitalized patients with moderate to severe COVID-19 pneumonia. Int. J. Infect. Dis. 102, 538-543. https://doi.org/10.1016/j. ijid.2020.11.008 (2021).

35. Lou, Y. et al. Clinical outcomes and plasma concentrations of Baloxavir Marboxil and Favipiravir in COVID-19 patients: An exploratory randomized, controlled trial. Eur. J. Pharmaceut. Sci. 157, 105631. https://doi.org/10.1016/j.ejps.2020.105631 (2020).

36. Udwadia, Z. F. et al. Efficacy and safety of Favipiravir, an oral RNA-dependent RNA polymerase inhibitor, in mild-to-moderate COVID-19: A randomized, comparative, open-label, multicenter, phase 3 clinical trial. Int. J. Infect. Dis.(IJID) 103, 62-71. https:// doi.org/10.1016/j.ijid.2020.11.142 (2020).

37. Zhao, H. et al. Tocilizumab combined with Favipiravir in the treatment of COVID-19: A multicenter trial in a small sample size. Biomed. Pharmacother. 133, https://doi.org/10.1016/j.biopha.2020.110825 (2021).

38. Erdem, H. et al. Treatment of SARS-cov-2 pneumonia with Favipiravir: Early results from the Ege University cohort, Turkey. Turk. J. Med. Sci. (2020).

39. Pérez-García, A. et al. A Randomized, Controlled Study on the Safety and Efficacy of Maraviroc and/or Favipiravir vs Currently Used Therapy in Severe COVID-19 Adults. "COMVIVIR" Trial. (2020).

40. Shrestha, D. B. et al. Favipiravir versus other antiviral or standard of care for COVID-19 treatment: A rapid systematic review and meta-analysis. Virol J. 17, 141-141. https://doi.org/10.1186/s12985-020-01412-z (2020). 
41. Pilkington, V., Pepperrell, T. \& Hill, A. A review of the safety of Favipiravir-A potential treatment in the COVID-19 pandemic?. J. Virus Erad. 6, 45-51. https://doi.org/10.1016/S2055-6640(20)30016-9 (2020).

42. Malvy, D., Taburet, A.-M., de Lamballerie, X., Mentre, F. \& Extramiana, F. The safety profile of Favipiravir should not be the first argument to suspend its evaluation in viral hemorrhagic fevers. PLoS Negl. Trop. Dis. 14, e0008259 (2020).

43. Noda, A., Shirai, T., Nakajima, H. Case Report Two Cases of COVID-19 Pneumonia Including Use of Favipiravir. 1-6 . Available at: http://www.kansensho.or.jp/uploads/files/topics/2019ncov/covid19_casereport_en_200408_2.pdf. (2021). Accessed 21 Aug 2020.

\section{Author contributions}

M.A.-Z.: conceptualization, methodology, formal Analysis, writing, validation, software, project administration, supervision; S.H.: methodology, formal Analysis, writing, validation, software; B.A.: methodology, data curation, writing; F.H.: methodology, data curation, writing; M.F.: methodology, data curation, writing; R.M.: supervision, writing, review and editing. All authors approve the manuscript before submission.

\section{Competing interests}

The authors declare no competing interests.

\section{Additional information}

Supplementary Information The online version contains supplementary material available at https://doi.org/ 10.1038/s41598-021-90551-6.

Correspondence and requests for materials should be addressed to M.A.-Z.

Reprints and permissions information is available at www.nature.com/reprints.

Publisher's note Springer Nature remains neutral with regard to jurisdictional claims in published maps and institutional affiliations.

(c) (i) Open Access This article is licensed under a Creative Commons Attribution 4.0 International License, which permits use, sharing, adaptation, distribution and reproduction in any medium or format, as long as you give appropriate credit to the original author(s) and the source, provide a link to the Creative Commons licence, and indicate if changes were made. The images or other third party material in this article are included in the article's Creative Commons licence, unless indicated otherwise in a credit line to the material. If material is not included in the article's Creative Commons licence and your intended use is not permitted by statutory regulation or exceeds the permitted use, you will need to obtain permission directly from the copyright holder. To view a copy of this licence, visit http://creativecommons.org/licenses/by/4.0/.

(C) The Author(s) 2021 